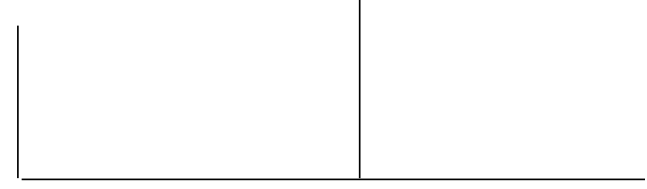

Rev. Latinoam. Psicopat. Fund., VIII, 1, 96-108

\title{
L'habiter ou le sentiment du chez soi
}

\author{
Rajaa Stitou
}

Il s'agit ici d'une contribution à un travail de réflexion clinique sur les incidences de l'habiter et du sentiment de chez soi. Ces incidences à travers lesquelles se manifeste tout un bouleversement de l'unité illusoire mais nécessaire dans laquelle se loge l'identité, s'exacerbe en terre étrangère au point d'exposer certains sujets expatriés à des risques de défaillance qui les empêchent d'investir de nouveaux liens, de se soutenir dans le monde. L'expérience clinique nous montre que l'habiter comme l'exil ne sont pas réductibles aux seules considérations territoriales et ethniques. Ils concernent chaque sujet dans son rapport à l'inconnu de son origine, de son appartenance et de son destin. L'expatriation ne fait que réactualiser cette épreuve de l'inconnu que tout un chacun peut élaborer en fonction de son histoire. Elle nous amène par ailleurs à reconsidérer la difficulté de penser l'articulation entre le sujet et le collectif, entre les processus psychique et le fait culturel.

Mots clés: Habiter, exil, étranger, sentiment d'identité, interculturel 
Le travail que je me propose d'engager ici est issu de ma pratique clinique auprès de sujets provenant d'autres cultures dans le cadre d'un centre médico-psychologique. Ce qui a attiré mon attention avec acuité, ce sont les plaintes répétitives de ces patients insistant parfois de manière dramatique sur cette difficulté d'habiterhors de chez soi à travers laquelle se manifeste tout un bouleversement de l'unité illusoire mais nécessaire dans laquelle se loge l'identité. Ces plaintes nous donnent à entendre que le sentiment du chez soi, qui permet de se mouvoir sans craindre l'errance, est parfois menacé à tel point que la terre étrangère n'est plus espace d'habitat mais lieu de l'angoisse et du mal vivre.

Afin de lutter contre ce malaise de l'habiter, certains sujets tentent de reconstituer chez eux un cadre qui leur rappelle celui du pays natal, et se raccrochent à une accumulation d'objets, meubles, tapis... afin d'introduire une présence familière. D'autres se font construire ou construisent eux-mêmes une maison dans le pays "d'origine", maison qui sera le garant de la permanence du lieu quitté, qui sera le lieu idéalisé d'un retour imaginaire toujours différé. Cette maison tant rêvée, souvent inhabitée, se transforme en mausolée ou en lieu de pèlerinage ritualisé durant les congés.

Cette atteinte du sentiment de chez soi se renforce particulièrement avec l'expatriation et se traduit par des symptômes d'appel témoignant d'une impossibilité de certains sujets d'investir de nouveaux liens, de se soutenir dans le monde, de perpétuer la généalogie ; ou de mettre au monde un enfant dans un lieu qui, selon l'expression de F. Benslama (1992) "ne fait pas monde". L'expérience clinique nous montre que ce n'est pas l'expatriation qui génère une telle souffrance, mais c'est ce qu'elle ravive en fonction de l'histoire du sujet. C'est aussi ce à quoi elle renvoie: à savoir cet exil originaire, fondateur, que la psychanalyse situe du côté du mythe et qui fait de tout sujet un étranger à lui-même du fait de sa division, de son incomplétude et de sa mortalité. Autrement dit, exil et expatriation sont ici à distinguer Ainsi que je l'ai montré par ailleurs (Stitou, 1997), si l'expatriation implique une rupture dans la réalité géographique, l'exil renvoie tout être parlant à l'indétermination de son origine, de son appartenance et de son destin. L'exil comme l'habiter évoque aussi ce lieu de mémoire, celui de la géographie psychique, là où frontières et 
franchissement concernent la relation de tout un chacun à l'inconnu dont il est porteur.

Voilà ce qui nous amène à reconsidérer ce qu'est le fait d'habiter, et à interroger ce qui lie le sujet au lieu.

\section{L'habiter et la nostalgie du lieu perdu}

Habiter n'est pas seulement occuper l'espace comme nous le laisserait entendre cette conception technocratique de l'architecture et de l'aménagement urbain.

Dans un texte intitulé "Bâtir, habiter, penser", M. Heidegger (1958) se livre à une analyse de "l'habiter" fondée sur l'étymologie allemande du mot "Bauen" (habiter). Il oppose loger et habiter ainsi que construire et bâtir. "Bâtir est déjà de lui même, habiter", nous dit-il, rapprochant l'être de l'habiter de celui de bâtir. Leur étymologie dans le haut allemand rejoint le verbe être (ich bin: je suis).

L'homme, selon Heidegger n'est tel que pour autant qu'il habite, bien que le sens propre de ce mot se soit affadi derrière un ensemble de significations.

Habiter renvoie en fait à un autre terme "Wumian" qui signifie demeurer en paix. Demeurer en paix dans ce qui nous est proche, dans ce qui est libre, "dans ce qui ménage toute chose dans son être" (ibid.), dans ce qui permet à l'homme de vivre au milieu des éléments, des choses en préservant leur être.

Le rapport de l'homme à l'espace, à travers des lieux réside dans l'habitation, c'est à dire dans le fait de pouvoir inscrire, comme dans les maisons paysannes de la forêt Noire l'existence humaine dans ses moments cruciaux, la naissance, la mort, dans un espace qui leur donne sens.

La conception Heideggerienne de l'habiter, à savoir ce qui rassemble, ce qui procure paix et sérénité, peut être rapprochée de cette aire d'expérience, cette aire transitionnelle entre l'enfant et sa mère, aire de jeu, lieu d'une expérience culturelle, définit par Winnicott (1971).

Pour l'auteur, ce serait dans ce lieu que nous nous situons lorsque nous ne sommes ni dedans, dans nos pensées, ni dehors, auprès d'un objet.

C'est dans ce lieu que le sujet peut s'inscrire dans un rapport au monde et aux choses. Ce lieu qui évoque souvent le "natal" n'est pas définissable comme une simple localité ou un territoire. "Il est ce lieu symbolique que personne ne peut ni me voler ni m'acheter. Il est une pure représentation que l'on emporte avec soi et que beaucoup de familles concrétisent dans un meuble, un tableau, un langage" écrit B. Salignon (1992).

Mais il relève avant tout de l'expérience, il correspond à l'intime. C'est ce dans quoi le sujet peut se rassembler, se ressourcer pour poursuivre sa route. 
"Le natal, nous dit encore B. Salignon (1992), peut être pensé comme le premier chez soi qui ne prend son sens qu'après coup, que rétroactivement; le natal est ce point de retour dont Ulysse est le représentant fondateur".

Le lieu qui renvoie au secret des origines, qui se situe non seulement du côté de ce qui est mémorisable, mais du côté de l'être, échappe à la mise en forme communicable, sinon par le moyen de l'oeuvre d'art. Mais ce lieu n'est pas un trésor à posséder, à détenir jalousement. En tant que lieu/hors lieu, il est à "trouvercréer" (Winnicott) en permanence. Il est aussi à partager.

De nombreux textes littéraires des siècles passés évoquent la rencontre d'un "pays" (mot qui se traduit dans le langage actuel par compatriote et qui fait appel à l'idée abstraite de patrie) comme un événement heureux dans le parcours d'un voyageur. Cette rencontre permet le partage, au delà de l'évènementiel et des signes culturels, de cette part d'indicible.

Ce partage est peut être ce qui fait défaut à certains sujets expatriés. Malgré leur tentative de recréer commerce et lieu de culte comme dans le pays des origines, malgré leur regroupement entre semblable, le partage est en faillite car il échoue à prendre en compte la différence et la singularité de l'expérience. En recherchant le retour au même, en se logeant dans les mêmes lieux entre compatriotes, le groupe des personnes "exipatriées" se constitue en ghettos, en espace clos dans une atmosphère trouble. Ce repli sur soi est parfois nourri par le regard rejetant de l'autochtone, qui dans une sorte de réplique symétrique, est lui aussi à la recherche de la mêmeté à travers la nostalgie d'un espace figé dans l'idéalisation, objet d'une défense acharnée contre toute atteinte, toute intrusion. Le repli sur soi est contraire à l'habiter car il induit un temps "mort", privé de nouveauté, et un vécu de l'espace oppressant ou défensif; sentiment souvent exprimé à propos des cités de banlieue.

La dégradation des équipements collectifs est souvent la conséquence de cet environnement vécu comme peu accueillant. La peur de l'Autre conduit à l'enfermement dans son espace propre ou, au contraire à l'errance - égarement dans la cité où se manifeste l'impossibilité d'habiter, d'avoir lieu au sens d'avoir un "site" pour déposer quelque chose de son être.

La maison évoque aussi l'enfance que l'on porte avec soi. C'est ce qui nous protège de l'obscurité. Quand un enfant se met à dessiner, n'est-ce pas souvent une maison qu'il esquisse?

Cela renvoie, écrit J-P. Martineau (1995) à “ toutes ces expériences précoces d'enveloppement, d'attachement, de portance" qui donnent lieu à "une conciliation, un métissage des étrangetés", à travers lesquelles s'instaure un lien de confiance Cette confiance qui est à situer du côté de la "fides", voire à la foi, ainsi que l'indique l'étymologie c'est ce que nous pouvons rattacher à cette acceptation originelle que Freud nomme "Bejahung", terme repris par Lacan (1966) qui lui a 
donné le sens d'un "dire oui au symbolique", d'un accord tacite, d'une prise de position de l'être qui accepte la dépossession de la jouissance de la Chose maternelle, ce qui constitue la base d'un rapport au monde permettant à la pensée de s'exercer.

"Habiter (du grec: oïken), nous dit encore J-P. Martineau (1995), s'articule avec porter (du grec: phorein) qui renvoie au corps maternel".

Mais toute femme en tant que mère potentielle n'est elle pas celle qui habite le vide? Est-ce pour cela que, dit-on, elle est plus sensible aux déménagements.

L'expérience clinique nous montre en effet que la problématique de l'habiter se fait plus intense chez les femmes qui vont donner naissance à un enfant ou qui ont du mal à concevoir, comme si le lieu d'accueil n'était pas vécu comme un lieu qui permet de donner lieu. Cette façon de se retrouver sans abri malgré le fait d'être logé est souvent vécue avec violence. Le langage populaire traduit bien cette dimension à travers l'expression: "ça déménage". Le fait de se sentir exclu dans sa propre maison ne renvoie-t-il pas aux premiers liens mais aussi aux premières séparations qui fondent tout sujet?

Autrement dit, la maison renvoie certes au corps de la mère, mais ne s'y confond pas. Car c'est aussi avec son désir que le sujet habite une maison, c'està-dire avec son manque à être, manque que le "Nom du père" porte en lui comme signifiant. C'est ce manque qui introduit la distance nécessaire qui régule le rapport du sujet à lui-même et au monde. Supprimer cette distance serait anéantir le sujet lui-même.

Par-delà sa dimension rationalisable, une maison ouvre toujours sur autre chose. Elle ouvre aussi sur cette immémorialité de l'ailleurs (à savoir l'inconscient) que tout un chacun transporte avec soi. Pour Freud, la maison est la métaphore de l'appareil psychique. A travers elle se dessine un dedans et un dehors. Ne dit-on pas d'une maison qu'elle a une âme? Ne parle-t-on pas également de "notre intérieur" pour dire "notre maison"?

La maison évoque l'intériorité, mais elle ouvre aussi sur un lieu de passage, une coupure-lien que réunit la dimension symbolique. La maison n'est donc pas un refuge clos. Elle garde un lien avec le dehors, lien à travers lequel se présentifie la dialectique de l'enclore et de l'éclore.

N'est-ce pas la mise à mal de cette dialectique qui porte atteinte au sentiment du chez soi, empêchant par là même le sujet d'affronter l'épreuve de l'inconnu? C'est alors que surgit le rejet du tout Autre.

Dans ce cas, l'exil n'est pas vécu comme quelque chose de constructif, mais comme une dispersion, comme une menace qui n'autorise plus le sujet à habiter son désir.

Pourrait-on dire que si les sujets expatriés se logent, certains d'entre eux ont du mal à habiter, à faire que leur domicile soit le lieu qui rassemble les éléments 
et les humains, qui organise le rapport à l'espace et au temps. Ainsi, depuis le décès de son fils aîné, Ahmed n'a qu'une idée en tête, construire une maison au Maroc, à côté du cimetière dans lequel est enterré son fils. "Ibni" (mon fils) et "l'bni" (le fait de bâtir une maison) seront les deux signifiants dont l'homophonie résonnera tout au long de l'entretien (lors de notre rencontre au CMPP), instaurant une sorte de confusion entre la maison à construire et le fils mort, entre le fils mort et le fils vivant qui a du mal à investir la scolarité.

"Je veux construire une maison dans mon pays pour ne pas perdre mon âme. Je veux donner un lieu à mes enfants où ils pourront se sentir chez eux. Je veux qu'ils aient un intérieur, ce que je n'ai pas eu la chance d'avoir moi. Après toutes ces années de sacrifice en terre étrangère, je veux que mes enfants se souviennent de moi après ma mort comme d'un père qui a fait quelque chose pour eux. J'espère 'inch'allah' que je mourrais dans mon pays".

La perte de son fils vient, pour Ahmed, mettre à nu des blessures anciennes qui appellent des rêves de retour à une origine mythique. Mais l'achat plus tard d'une maison au Maroc n'apaise pas pour autant la douleur d'Ahmed face à ce hors sens que convoque la séparation et qui exacerbe ce que ravive l'épreuve de l'extranéité. Car la douleur d'exister ne concerne pas seulement la division entre deux pays et deux cultures, mais le renvoie à sa propre division subjective, à son incomplétude et à sa mortalité. Elle le renvoie également à ce qu'il en est de sa généalogie, de ses identifications...

De même la nostalgie du lieu ouvre sur une pluralité de lieux: réel, imaginaire, symbolique. Elle concerne toujours cette Autre scène qu'est l'inconscient, à travers laquelle se déploie un espace psychique marqué par la subjectivité et les références fondatrices du sujet, par lesquelles l'homme s'inscrit dans un héritage qui le précède et qu'il tente de transmettre à son tour. Par conséquent, il serait ici réducteur de confondre le lieu avec la terre perdue, car ce n'est pas seulement une maison réelle que recherche Ahmed. Ce qu'il recherche à travers son deuil difficile à effectuer, c'est à retrouver des repères symboliques, afin de se préserver contre l'insoutenable du réel de la mort. C'est en renouant avec les "signifiants" qui le constituent et en élaborant ce qui fait manque au plus intime de lui-même qu'il peut s'autoriser à habiter sa maison, avec son désirs et sa subjectivité.

Mais pour que cet espace soit habitable, c'est-à-dire vivable, pour qu'il ne se transforme pas en tombeau, il faut qu'il soit capable d'ouvrir sur d'autres lieux, sur d'autres liens. C'est ainsi qu'il préserve du non-lieu et de l'égarement.

Le travail entrepris avec Ahmed a consisté ni à congédier, ni à fétichiser sa différence culturelle. Il a consisté à l'aider non pas à retrouver son lieu d'origine que nul ne peut s'approprier, mais à se réconcilier avec sa mémoire en y impliquant sa position de sujet. C'est ainsi que peut s'ouvrir la voie d'un travail de 
métaphorisation à partir de laquelle le sujet consent à réorganiser son passé en histoire.

Ce fragment clinique nous incite à réfléchir sur les pièges de certaines pratiques dites "interculturelles". En effet, le rapport à "l'étrangement culturel" n'a pas été indemne des différentes manières de penser des différents courants qui se sont affirmés en moins d'un siècle. C'est à cette question qui nous amène à reconsidérer la difficile articulation entre le singulier et le collectif, entre le psychique et le culturel, que je propose à présent de laisser place.

\section{“L'étrangement culturel”: quelques éléments historiques et théoriques}

Nous laisserons de côté les récits des anciens, ou des voyageurs de la Renaissance où l'observation précise côtoie le merveilleux comme si ce dernier était une figure obligée de l'étrangéité.

C'est seulement au tournant de l'âge classique, moment de la naissance de la subjectivité, précédé en peinture par l'apparition de la perspective ${ }^{1}$ que les premières manifestations subjectives du contact avec l'étranger vont faire l'objet d'une approche rationnelle. Johannes HOFER, médecin de Bâle, témoignera d'un intérêt particulier pour la nostalgie, mot qu'il construit à partir des vocables grecs "nostos", signifiant le retour et "algos", la douleur.

Ce mot introduisait dans la langue savante des facultés de médecine l'expression populaire "d'Heimweh", mal du pays, caractérisant l'état de soldats que l'éloignement de leur région ou de leur famille amenaient à dépérir, au point qu'il était parfois nécessaire de les renvoyer dans leurs foyers.

A ce moment-là, le "choc de l'étranger" est apprécié dans sa dimension psychique et des traitements moraux sont préconisés: permission pour retourner au pays ou rapatriement, attitude paternelle de la hiérarchie.

Cette approche sera toutefois contrariée par le développement de l'anatomopathologie à la suite de la découverte des lésions cérébrales accompagnant la paralysie générale. Le recours au physiologique comme cause ultime des troubles psychiques au XIX siècle rabattra la nostalgie du côté de l'organique. Elle n'en restera pas moins la première approche "interculturelle" soulignant l'effet et la prégnance du rapport à l'étranger dans les sentiments de destitution subjective et les dérégulations somatiques que peut traverser un humain en situation

1. Où c'est l'œil de l'observateur matérialisant la place du sujet qui devient le centre de référence. 
d'expatriation. Avec Hugo, Lamartine et Musset, elle deviendra un thème littéraire marquant la génération de jeunes gens de 1830 pris entre le regret du monde ancien ou de l'épopée napoléonienne et les montées des nationalismes, les révolutions qui vont survenir.

Mais le développement technique et économique; voyages et expéditions d'exploration ou de conquête, vont ouvrir l'Occident sur des pays lointains et entraîner dans divers secteurs de la vie sociale et de la création artistique ce qui a été appelé l'orientalisme.

Cet intérêt pour l'Orient entraînant une ouverture vers sa culture se double d'un questionnement inverse sur l'universalité des classifications que la psychiatrie occidentale est en train d'établir. A travers cette question, c'est encore une fois la définition de l'humain qui est en jeu: existe-t-il des primitifs et des être évolués? Se repose aussi la question des rapports entre nature et culture, qui trouvera un développement à travers le culturalisme.

Cette époque de conquêtes coloniales, est aussi celle où la France va chercher à établir, dans le Maghreb plus particulièrement, un système de santé mentale, calqué sur la métropole en Algérie, plus souple et tenant davantage compte des institutions locales au Maroc.

S'élabore donc une psychiatrie spécifique sur le modèle de la médecine découvrant des maladies exotiques, dans une démarche qui fait partie de la "mission civilisatrice" de la France.

Ainsi que le montre J. Bennani (1996) dans son historique, c'est l'école d'Alger avec A. Porot qui va marquer toute une tradition psychiatrique dans le Maghreb en abordant, dans un esprit qui se voulait scientifique, et à partir des théories de E. Dupré concernant les constitutions, les phénomènes morbides.

Les titres de quelques articles de A. Porot résument cette démarche qui tend à passer du fait pathologique singulier à une psychiatrisation de la culture, la situant ainsi dans le champ de l'anormalité. "L'impulsivité criminelle chez le Nordafricain algérien" en 1932, "Le primitivisme des indigènes nord-africains" en 1939, écrit en collaboration avec J. Sutter, montrent l'impact du fait colonial sur la démarche scientifique à ce moment là.

Au Maroc, P. Sérieux, auteur du "Délire d'interprétation" (1909), est mandaté par le gouvernement pour étudier la manière dont un service psychiatrique pourrait s'implanter.

Les autorités françaises tentèrent dans ce pays de restaurer des institutions, les "maristânes" qui, depuis le XV siècle, hébergeaient des malades mentaux. Simultanément, des praticiens formés à Alger mais aussi des psychiatres ayant eu contact avec la psychanalyse, par l'intermédiaire de R. Laforgue réfugié au Maroc pendant la deuxième guerre mondiale, faisaient de la psychiatrie marocaine un ensemble moins monolithique. 
Bien que Laforgue (1954), ait développé des idées ségrégatives sur un "super-ego culturel", les idées psychanalytiques avaient tout de même suscité chez ses élèves des initiatives dans le sens d'une désincarcération du malade et d'une plus grande écoute.

En résumé, la psychiatrie coloniale a tenté, malgré quelques exceptions, de plaquer des modèles dans une visée scientifique objectivante sans tenir compte des soubassements subjectifs de tel ou tel symptôme ou de telle ou telle conduite, mais en réduisant au contraire le sujet aux formes culturelles de son symptôme, en lui confisquant sa parole.

Cette démarche se rapproche par sa dimension réductrice de l'abord ethnologique culturaliste qui définit le sujet humain comme pur produit des conditions culturelles qui sont celles de son environnement. On connaît en particulier les conclusions de Margaret Mead (1963) comparant deux populations voisines de Nouvelle-Guinée, l'une prônant la douceur et la tendresse, l'autre la violence.

Mais ces théories culturalistes n'ont pas manqué d'être vivement critiquées, d'abord en raison de la démarche empirique qui les sous-tend, réduisant les comportements à des types dont la construction est fortement marquée par l'idéologie des chercheurs. Ne viendraient-elles pas en outre soutenir une orientation de la pensée politique anglo-saxonne qui a inspiré leur approche, prônant la coexistence de communautés différentes, où la norme serait celle d'un "chacun chez soi".

Dans son appel à l'anthropologie Freud (1984) a certes pris appui sur les conceptualisations évolutionnistes de son époque, afin d'établir des "concordances dans la vie psychique des sauvages et des névrosés" (sous titre de "Totem et tabou").

Toutefois, sa démarche ne s'arrête pas à ces préjugés et ouvre au contraire un passage en explorant les rapports de l'homme au symbolique comme elle l'avait fait pour les névrosés.

C'était-là, bien au-delà des spécificités culturelles retrouver une constante du fait humain qui avait été déniée par les culturalistes. Tout être humain est avant tout un être mortel sexué et parlant.

Géza Roheim (1950), à la suite de Freud et à la lumière de la théorie psychanalytique, étudiera dans une perspective ontogénétique la façon dont les différentes cultures traitent le rapport à l'origine, à la mort, à la différence des sexes.

De son côté Georges Devereux (1970), fondateur de l'ethnopsychiatrie tentera d'articuler démarche analytique et anthropologique à partir d'une méthode qui consiste à utiliser les deux référentiels successivement. Il distingue deux types d'inconscient: l'inconscient idiosyncrasique (l'inconscient du sujet) et 
l'inconscient ethnique, c'est à dire "cette partie de son inconscient que le sujet a en commun avec tous les membres de sa culture".

C'est à partir de cet inconscient ethnique que Deveureux va créer toute une catégorie nosographique qu'il dénomme "désordre sacré" (le chamanisme). Avec sa théorie culturelle de l'inconscient, cet auteur qui a eu le mérite d'introduire une nouvelle épistémologie en sciences du comportement grâce à sa prise en compte de la subjectivité de l'observateur ouvre cependant la voie vers le piège qu'il a luimême dénoncé, à savoir l'ethnocentrisme. C'est sur la méthode de Devereux que s'appuiera la nouvelle ethnopsychiatrie afin d'aménager un cadre thérapeutique spécifique de la problématique des migrants. L'une des règles fondamentales de ce cadre est de prendre en charge les patients étrangers en fonction des critères de leur ethnie et de leur langue en reconstituant s'il le faut une thérapie traditionnelle, telle que la pratique le shaman ou le marabout.

Or, en réduisant le sujet à sa différence ethnique, un tel modèle ne risquet-il pas d'enfermer la personne étrangère dans une identité close sur elle-même, et de faire taire l'expérience propre de chacun.

Cette démarche qui montre certes la fécondité d'un rapprochement entre différents champs conceptuels dans la rencontre avec des sujets provenant d'autres cultures a donc aussi ses limites à travers le risque d'un confusionnisme qu'elle peut comporter: car en effet comment peut-on tenir tantôt la positon du psychanalyste, tantôt la position de l'anthropologue ou de l'ethnologue sans être dans le clivage ou la confusion?

Enrichir la pratique analytique de connaissances sur le rapport de l'homme au symbolique dans les différentes cultures ne peut qu'être positif, à condition de ne pas fétichiser la différence en "ghettoïsant" des sujets qui ont parfois du mal à investir une nouvelle réalité. Que des mythes et des croyances issues d'ailleurs mettent en forme ou sous-tendent des discours de souffrance, c'est tout l'intérêt d'une approche dite "interculturelle" de le mettre en lumière. Cela n'implique pas pour autant que le thérapeute tente de se transformer en shaman ou en thérapeute traditionnel pour accueillir des patients étrangers.

Nous remarquons donc que, derrière ces différentes modélisations théoriques, se profile l'enjeu et la difficulté de penser l'articulation entre le sujet et le collectif, entre les processus psychiques et le fait culturel.

Ainsi que je l'ai montré par ailleurs (1997) cette difficulté provient du clivage qui disjoint le sujet de la réalité qui l'entoure, soit en ne l'appréhendant qu'à travers le symptôme supposé social et/ou culturel, soit en mettant l'accent sur un psychisme fermé sur lui-même. Autrement dit, il y aurait d'un côté le sujet et d'un autre côté le social; d'un côté le symptôme inhérent au sujet et d'un autre côté le symptôme lié à la culture ou à la civilisation. 
Afin de sortir de ces clivages, il convient de redéfinir ce que nous entendons par symptôme. Tout symptôme est à l'interface du sujet singulier [dans sa tentative de signifier quelque chose de la vérité de son être], et du discours social. En tant qu'effet de l'autre, il combine à la fois le prescrit et le proscrit. Les formes du symptôme ont donc le plus étroit rapport avec les modalités d'expression dans un lieu et un moment historique donné. Le symptôme exprime ainsi un sujet dans le champ du collectif à travers l'émergence du refoulé, de l'indicible (R. Stitou, 1997, p. 25).

La rencontre de sujets provenant d'autres cultures nous permet donc d'interroger nos outils conceptuels, questionnement qui incarne le mouvement propre à la clinique qui, dans sa visée non clôturante et dans son souci du détail et de la valeur qu'il prend dans la parole même du patient, maintient un regard et une écoute de la dimension infiniment singulière du sujet pris dans les variations socioculturelles et historiques. La prise en compte des codifications culturelles s'avère donc nécessaire, mais à condition de ne pas confondre la culture qui n'existe pas indépendamment du sujet et le culturalisme qui consiste à se figer dans des caractéristiques imaginaires, exotiques, au détriment de la dimension symbolique, et qui par conséquent renforce chez l'autre la méconnaîssance de sa propre division subjective.

\section{Références}

Benslama, F. L'enfant et le lieu. Cahiers Intersignes, 3, p. 51-8, 1992.

Bennani, J. La psychanalyse au pays des saints. Casablanca: Le Fennec, 1996.

Devereux, G. Essai d'ethnopsychiatrie générale. Paris: Gallimard, 1970.

Freud, S. (1912). Totem et tabou. Paris: Payot, 1984.

(1925). La négation. Résultats, idées, problèmes TII. Paris: PUF, 1985.

HeIDEgGer, M. Bâtir, habiter, penser. Essais et conférences. Paris: Gallimard, 1958. p. 170-93.

IGGER, M. Introduction à la psychopathologie Marocaine. Maroc Médical, 350, p. $1310-30,1955$.

LaCAN, J. Commentaire parlé de Freud sur la "Verneinung" de Freud par J. Hyppolite. In: Ecrits. Paris: Seuil, 1966. p. 879-88.

LAfORgue, R. Le super-ego individuel et collectif. Au delà du scientisme. Genève: Mont-Blanc, 1954.

Martineau, J.-P. (1955). Anthropologie clinique de l'habiter. Homo, n. XXXIV, p. 155-224, 1995.

Mead, M. Moeurs et sexualité en Océanie. Paris: Plon, 1963.

Nathan,T. L'influence qui guérit. Paris: Odile Jacob, 1994. 
Roнеiм, G. Psychanalyse et anthropologie. Paris: Gallimard, 1950.

SAlignon, B. Qu'est-ce qu'habiter? Nice: Z'éditions, 1992.

Stitou, R. Universalité et singularité de l'exil. Psychologie clinique, 3, p. 13-20, 1997.

WinNicotT, D. W. Jeu et réalité. Paris: Gallimard, 1971.

\section{Résumé}

Trata-se de uma contribuição a um trabalho de reflexão clínica sobre as incidências do habitar e do sentimento de "lar". Estas incidências, por meio das quais se manifesta toda uma reviravolta da unidade ilusória, mas necessária, na qual habita a identidade, exacerbam-se em terra estrangeira a ponto de expor certos sujeitos expatriados a riscos de fragilidade que os impedem de investir novos vínculos, de se sustentar no mundo. A experiência clínica demonstra que o habitar, assim como o exílio, não se reduzem unicamente a considerações territoriais e étnicas. Eles dizem respeito a cada indivíduo em sua relação com o que há de desconhecido em suas origens, em seu pertencimento e em seu destino. A expatriação só vem reatualizar esta provação do desconhecido que qualquer indivíduo pode elaborar em função de sua história. Ela nos leva, além disso, a reconsiderar a dificuldade de pensar a articulação entre o sujeito e o coletivo, entre os processos psíquicos e o fato cultural.

Palavras-chave: Habitar, exílio, estrangeiro, sentimento de identidade, intercultural

El presente estudio es una contribución a un trabajo de reflexión clínica sobre las incidencias de habitar un lugar y del sentimiento de hogar. Estas incidencias, por las cuales se manifiesta una necesaria conmoción en la unidad ilusoria de la identidad, se exacerban en tierra extraña hasta tal punto que algunos sujetos expatriados están expuestos a riesgos de desasosiego que los coartan para trabar nuevos lazos e para sustentarse en el mundo. La experiencia clínica nos enseña que tanto habitar como el exilio no se reducen a consideraciones territoriales y étnicas. Cada sujeto los vive en su relación con lo desconocido a su origen, su pertenencia y su destino. La expatriación es el factor que reactualiza el reto de lo desconocido que cada cual puede elaborar en función de su historia.

La expatriación, por su parte, nos lleva a reconsiderar la dificultad de pensar la articulación entre el sujeto y lo colectivo, entre el proceso psíquico y el hecho cultural.

Palabras claves: Habitar, exilio, extranjero, sentimiento de identidad, intercultural

This paper is meant as a contribution to the clinical reflection on the effects of dwelling, or living, and a feeling of "home". Through these effects, an entire (and necessary) revolution of illusory unity becomes evident, wherein lies identity. And they become even stronger in a foreign land to the point of exposing certain expatriated 
subjects to risks of fragility that prevent them from investing in new relationships and from sustaining themselves in the world. Clinical experience shows that dwelling, or living [somewhere], as well as exile, cannot be restricted exclusively to territorial and ethnic considerations. They concern the relationship of each subject with the strangeness of his origins, his family ties and his destiny. Expatriation merely accentuates this suffering of the unknown that any individual can organize according to his own history. It also leads us to reconsider the difficulty of understanding the articulation between the individual subject and the community, between mental process and cultural factors.

Key words: Dwelling, exile, foreigner, sense of identity, intercultural

Versão inicial recebida em abril de 2003

Versão revisada recebida em março de 2004 